\title{
Ein experimenteller Beitrag zur Theorie des elektrischen Widerstandes der Metalle
}

\author{
Von R. Nossek \\ Aus dem Physikalischen Institut der Bergakademie Clausthal \\ (Z. Naturforschg. 16 a, 1162-1172 [1961] ; eingegangen am 27. März 1961)
}

\begin{abstract}
Es wird eine Meßzelle beschrieben, mit der bei einem Druck von $p \leq 5 \cdot 10^{-9}$ Torr der elektrische Widerstand dünner, auf einem Glasträger aufgedampfter Alkalimetallschichten bei $90{ }^{\circ} \mathrm{K}$ und $60^{\circ} \mathrm{K}$ in Abhängigkeit von der Schichtdicke gemessen wurde. An denselben Schichten wurde auch ihre Thermospannung gegen Platin als Bezugsmetall in Abhängigkeit von der Schichtdicke gemessen. Aus dem experimentell ermittelten Verlauf des spezifischen elektrischen Widerstandes von Kalium, Rubidium und Cäsium als Funktion der Schichtdicke wurde die mittlere freie Weglänge der Leitungselektronen bestimmt und das Verhältnis der Leitungselektronen zur Zahl der Atome berechnet. An Schichten, deren Dicke größer als die mittlere freie Weglänge ist, wurde für die thermische Widerstandsänderung $\mathrm{d} \varrho / \mathrm{d} T$ der Wert des kompakten Metalles gefunden. Das bedeutet, daß in dünnen Schichten der Idealwiderstand genau so groß ist wie der im unendlich ausgedehnten Metall. Der höhere spezifische Widerstand dünner Aufdampfschichten wird nur durch die höhere Konzentration der Gitterfehler und durch die nicht mehr vernachlässigbare Wechselwirkung der Elektronen mit den Grenzflächen verursacht. Daher konnte die mittlere freie Weglänge $l_{\infty}$ in die beiden Anteile $l_{\mathrm{i}}$, die den Gitterschwingungen und $l_{\mathrm{r}}$, die den Gitterfehlern zuzuordnen sind, aufgespalten werden. Ferner konnte die Möglichkeit einer neuen Methode der Schichtdickebestimmung nachgewiesen werden. Aus dem Verlauf der Thermospannung als Funktion der Schichtdicke wurde die Abhängigkeit der mittleren freien Weglänge von der Energie der Leitungselektronen ermittelt. Die Voraussage der Bцоснschen Theorie: $l_{\mathrm{i}}=a E^{2}$ wird vom Experiment für Kalium und Rubidium bestätigt. Für Cäsium ergibt sich jedoch ein Exponent, der zwischen 6 und 9 liegt.
\end{abstract}

\section{Grundlagen und Durchführung der Messungen}

\section{Einführung}

In der Elektronentheorie der Metalle ${ }^{1,2}$ wird gezeigt, daß die dem Idealwiderstand zuzuordnende mittlere freie Weglänge der Leitungselektronen quadratisch von der Energie der Elektronen abhängt. Erstmals haben Justi, Kohler und Lautz ${ }^{3,4}$ versucht, diese Aussage der Theorie experimentell nachzuprüfen. Ihre an $\mathrm{Bi}$ und $\mathrm{Pb}$ durchgeführten Messungen bestätigten die Richtigkeit ihrer für diesen Zweck entwickelten Theorie. Leider war aber weder ihre Meßmethode ausreichend entwickelt noch war die Wahl der Metalle günstig, um einen zwingenden Schluß bezüglich der Energieabhängigkeit der mittleren freien Weglänge zu erlauben.

Spätere Messungen von Savornin und Couchet ${ }^{5}$ an $\mathrm{Al}$ und von Reimer ${ }^{6}$ an $\mathrm{Ta}, \mathrm{Sb}, \mathrm{Bi}, \mathrm{Fe}, \mathrm{Ni}, \mathrm{Co}$ und Al ließen sowohl die Theorie von Justi, Kohler und Lautz als auch die Blochsche Theorie des elek-

1 A. Sommerfeld u. H. Bethe, Elektronentheorie der Metalle, Handb. d. Phys. 24/2, 1933.

2 A. H. Wilson, The Theory of Metals, Univ. Press., Cambridge 1953 .

${ }^{3}$ E. Justi, M. Kohler u. G. Lautz, Naturwiss. 38, 475 [1951].

4 E. Justi, M. Kohler u. G. Lautz, Z. Naturforschg. 6 a, 456, 544 [1951]. trischen Widerstandes fraglich erscheinen. Bei den untersuchten Metallen konnte aber kaum erwartet werden, daß die in der Theorie des elektrischen Widerstandes gemachten Annahmen zutreffen. Diese Diskrepanz ließ es wünschenswert und reizvoll erscheinen die Alkalimetalle zu untersuchen, weil diese den theoretischen Annahmen am besten entsprechen.

\section{Die Meßmethode}

Alle Phänomene, die von der mittleren freien Weglänge der Leitungselektronen abhängen, zeigen bei dünnen Schichten oder Drähten ein vom unendlich ausgedehnten Metall abweichendes Verhalten, weil die Wechselwirkung der Elektronen mit den Grenzflächen gegenüber der im Innern des Metalles auftretenden Wechselwirkungen nicht mehr vernachlässigbar ist. Die Abhängigkeit der einzelnen Erscheinungen von der Dicke dünner Schichten wird quantitativ in der Theorie der Weglängeneffekte ${ }^{7,8}$ behandelt. Daraus kann man entnehmen, daß aus dem Verlauf der absoluten Thermokraft und des

5 J. Savornin u. G. Couchet, C. R. Acad. Sci., Paris 234, 1608 [1952].

6 L. Reimer, Z. Naturforschg. 12 a, 525 [1957].

7 H. Mayer, Physik dünner Schichten, Bd. II., Wiss. Verlagsges., Stuttgart 1955.

8 E. H. Sondheimer, Adv. Phys. 1, 1 [1952]. 
elektrischen Widerstandes als Funktion der Schichtdicke die mittlere freie Weglänge und ihre Abhängigkeit von der Energie der Leitungselektronen bestimmbar sind.

\section{Die Meßzelle}

Die Meßzelle mußte so gebaut sein, daß sowohl der elektrische Widerstand als auch die Thermospannung an denselben Schichten in Abhängigkeit von der Schichtdicke gemessen werden konnten. Sie wurde aus Hartglas (Duran 50) geblasen und ist in Abb. 1 schematisch

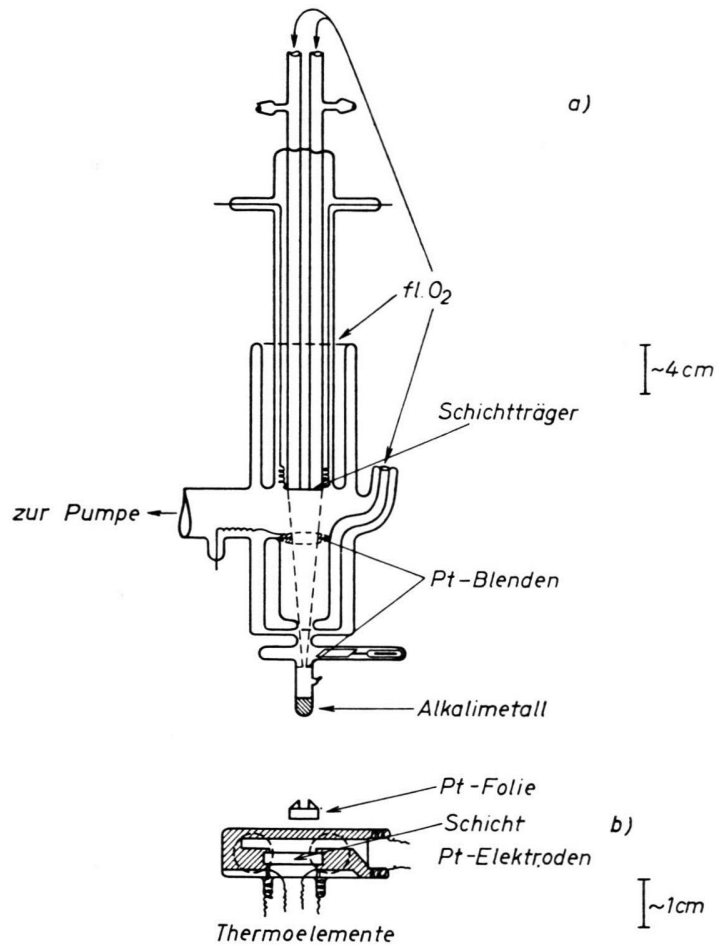

Abb. 1. a) Schematische Darstellung der Meßzelle; b) Schichtträger in Aufsicht.

gezeichnet. Ihren wichtigsten Teil bildet der Schichtträger, ein ebenes Glasplättchen, das als Boden auf zwei Kühlrohre, und als Brücke zwischen beiden, aufgeschmolzen war. Auf dieses Glasplättchen wurde die zu messende Schicht als Brücke zwischen den Platinelektroden in der in Abb. $1 \mathrm{~b}$ angedeuteten Weise aufgedampft. Durch geeignete Verbindungen der Elektroden mit der Meßanordnung konnten wahlweise gemessen werden:

1. Die Temperatur an den Berührungsstellen Schicht/ Platin,

9 Von der Fa. M e r c k, Darmstadt, bezogen.

10 In dankenswerter Weise von der $\mathrm{Hans}$ - $\mathrm{H}$ e in $\mathrm{r}$ i c h. Hütte GmbH., Langelsheim (Dr. MülLER) ausgeführt.
2. die differentielle Thermospannung der Schicht gegen Platin,

3. der elektrische Widerstand der Schicht.

Unmittelbar neben dem Schichtträger befand sich eine Pt-Folie, die zur Schichtdickebestimmung nach der LANGMUIR-Methode diente.

Glasplatte und Pt-Folie wurden in ein doppelwandiges Glasrohr eingebaut (Abb. $1 \mathrm{a}$ ), das mit flüssigem Sauerstoff beschickt werden konnte. Auf diese Weise wurden Schicht und Schichtträger weitgehend gegen die Wärmeeinstrahlung der Umgebung abgeschirmt. Die Schicht konnte daher ständig unter definierten und reproduzierbaren Temperaturverhältnissen gehalten werden.

Um die Kondensation vagabundierender Alkaliatome auf dem Schichtträger zu vermeiden, befand sich zwischen diesem und dem Atomstrahlofen ein zylinderförmiges Gefäß, in welches ebenfalls flüssiger Sauerstoff gefüllt werden konnte. Dieses Gefäß war an seinem unteren Ende als Blende ausgebildet, während die dem Schichtträger zugekehrte Seite eine aufgeschobene $\mathrm{Pt}$ Blende trug, welche die Geometrie der Schicht bestimmte. Dadurch wurde sichergestellt, daß die Schicht nur aus Atomen aufgebaut wurde, die aus der „punktförmigen“" $(\phi=0,75 \mathrm{~mm})$ untersten Atomstrahlblende kamen. Die an den unmittelbar darüber befindlichen Glaswänden kondensierten Atome konnten den Schichtträger nur über eine Reflexion an einer der tiefgekühlten Flächen dieses zylindrischen Gefäßes erreichen. An diesen kalten Wänden kondensierten sie und konnten weder den Aufbau der Schicht noch die Schichtdickebestimmung stören oder verfälschen.

\section{Die Vorbehandlung des Alkalimetalles}

Die Alkalimetalle $\mathrm{K}, \mathrm{Rb}$ und $\mathrm{Cs}$ wurden aus ihren Chloriden ${ }^{9}$ bei einem Druck von $p=3 \cdot 10^{-6}$ Torr durch Reduktion mit im Hochvakuum doppelt destilliertem Calcium gewonnen. Anschließend wurden sie bei einem Druck von $\sim 10^{-8}$ Torr in einer ausgeheizten Duranglasapparatur 4- bis 5-mal langsam mit einem elektrischen Ofen ohne Benutzung einer Gasflamme destilliert, in kleine Glasampullen eingefüllt und unter Vakuum abgeschmolzen. Eine Reinheitsprüfung ${ }^{10}$ der so behandelten Metalle ergab:

Kalium: $\quad \mathrm{Rb}, \mathrm{Cs}, \mathrm{Na}$ unter $0,01 \%$,

Rubidium: $0,2 \% \mathrm{Cs} ; 0,1 \% \mathrm{Na} ; \mathrm{K}$ unter $0,01 \%$, Caesium: $\quad 0,2 \% \mathrm{Rb} ; \quad 0,03 \% \mathrm{~K} ; \quad 0,02 \% \mathrm{Na}$.

Die für die Messung bestimmte Ampulle wurde an die Meßzelle so angeschmolzen, daß sie sich während des Ausheizens außerhalb des Ofens befand. Erst nachdem in der Meßzelle der Enddruck von $p \leqq 5 \cdot 10^{-9}$ Torr $^{11}$ erreicht worden war, wurde die Ampulle mit einer magnetisch bedienten Aufschlagvorrichtung geöffnet und das betreffende Alkalimetall wieder mittels elektrischer Heizung langsam in den Atomstrahlofen der Meßzelle über zwei Destillationsstufen eindestilliert.

11 Gemessen mit dem Ionisationsmanometer n. D. Alpert ${ }^{12,13}$. 12 D. Alpert, J. Appl. Phys. 24, 860 [1953].

13 D. Alpert u. R. S. Buritz, J. Appl. Phys. 25, 202 [1954]. 


\section{Die Schichtdickebestimmung}

Langmuir und Kingdon ${ }^{14,15}$ haben als erste beobachtet, daß Alkaliatome von der glühenden Oberfläche eines Metalles hoher Austrittsarbeit als Ionen verdampfen. In dieser Arbeit trafen die Alkaliatome auf die neben dem Schichtträger angebrachte $\mathrm{Pt}$-Folie (Abb. 1 a), die im Augenblick der Messung durch Stromdurchgang geglüht wurde. Der Strom der verdampfenden Ionen wurde über eine Sammelelektrode, an der eine negative Sättigungsspannung lag, gemessen. Aus der Stromstärke wurde unmittelbar die Zahl der sekundlich von der Pt-Oberfläche verdampfenden Ionen berechnet, die proportional der pro Sekunde auftreffenden Atome ist. Der Proportionalitätsfaktor, Ionisierungskoeffizient genannt, wird im gesamten älteren Schrifttum ${ }^{16}$ für $\mathrm{K}, \mathrm{Rb}$ und $\mathrm{Cs}$ an $\mathrm{W}$ und $\mathrm{Pt}$ mit eins angegeben, was jedoch nicht ausreichend begründet ist. Neuerdings haben $\mathrm{D}_{\mathrm{ATZ}}$ und $\mathrm{T}_{\mathrm{AYLOR}}{ }^{18}$ den Ionisierungskoeffizienten aller Alkalimetalle an W und $\mathrm{Pt}$ gemessen und Werte gefunden, die von eins abweichen.

In der vorliegenden Arbeit wurde die Dicke der aufgedampften Schichten aus dem gemessenen Ionenstrom unter folgenden Annahmen ermittelt:

1. Die von Datz und Taylor gemessenen Ionisierungskoeffizienten sind richtig.

2. Alle auf den Schichtträger auftreffenden Atome bleiben haften.

3. Die Dichte der Schicht ist gleich der des kompakten Metalles.

Die so berechnete Schichtdicke ist daher als eine „Massendicke" anzusehen. Sie ist sicherlich etwas kleiner als die geometrische Dicke, da ungetemperte Aufdampfschichten eine etwas geringere Dichte als das kompakte Metall haben.

\section{Der Meßvorgang}

Nachdem das Alkalimetall in den hierfür bestimmten Atomstrahlofen eindestilliert und das Restrohr der letzten Destillationsstufe abgeschmolzen worden war, stand die Apparatur zur Messung bereit. Der Meßvorgang spielte sich in folgender Reihenfolge $a b$ : In das unmittelbar über dem Atomstrahlofen befindliche zylindrische Gefäß wurde flüssiger Sauerstoff eingefüllt. Dann wurde der Atomstrahlofen durch ein darübergeschobenes aber in Abb. 1 nicht eingezeichnetes elektrisches Öfchen auf eine konstante, vorher bestimmte Temperatur gebracht.

Der Schichtträger wurde gleichzeitig mit Heizkörpern, die in die ihn tragenden Glasrohre hineinragten, auf etwa $400{ }^{\circ} \mathrm{C}$ erhitzt, um etwa wieder adsorbierte Atome oder Moleküle zu entfernen. Diese Behandlung wurde vor jedem Aufdampfen einer neuen Schicht während gleicher Zeiten wiederholt.

Sobald das Öfchen die gewünschte Temperaturkon$\operatorname{stanz}\left( \pm 0,1^{\circ} \mathrm{C}\right)$ erreicht hatte, wurde der den Schicht-

14 J. Langmuir u. K. H. Kingdon, Science (Lancaster, Pa.) 57, 58 [1923]; (Phys. Ber. 4, 1370 [1923]).

15 J. Langmuir u. K. H. Kingdon, Proc. Roy. Soc., Lond. A 107, 61 [1925]. träger umgebende Kühlmantel mit flüssiger Luft beschickt. Darauf wurde der Schieber über dem Atomstrahlofen zurückgezogen, der Atomstrahl auf die glühende Pt-Folie damit freigegeben und der Ionenstrom gemessen. Nun wurden die Heizkörper aus den Glasrohren entfernt und flüssiger Sauerstoff hineingefüllt.

Mit der Freigabe des Atomstrahls auf den auf $90^{\circ} \mathrm{K}$ gekühlten Schichtträger begann die Schicht als Brücke über die Pt-Elektroden zu wachsen. Da für kontrollierte Konstanz der Temperatur des Atomstrahlöfchens gesorgt war, wuchs die Schichtdicke proportional mit der Zeit. Sie war daher in jedem Augenblick bekannt. Hatte sie den gewünschten Wert erreicht, wurde der Atomstrahl gesperrt.

An dieser frisch aufgedampften Schicht wurde nun sowohl der elektrische Widerstand bei $90{ }^{\circ} \mathrm{K}$ und $60^{\circ} \mathrm{K}$ als auch die Thermospannung gemessen. Um eine Verfälschung der Meßwerte infolge von im Meßkreis möglicherweise vorhandener "wilder" Thermospannungen zu berücksichtigen, wurde bei den Messungen des elektrischen Widerstandes die Stromrichtung, bei denen der Thermospannung das Temperaturfeld umgekehrt.

Zunächst wurde der elektrische Widerstand gemessen. Darauf wurde die Temperatur der einen Kontaktstelle Schicht $/ \mathrm{Pt}$ erniedrigt, indem im zugehörigen Trägerrohr I der Druck durch Abpumpen auf 5-6 Torr gesenkt wurde. Die dabei entstandene Temperaturdifferenz wurde mit den hierfür vorgesehenen beiden Thermoelementen ermittelt und die aufgetretene Thermospannung der Schicht gegen Pt gemessen. Dann wurde der Druck in beiden Rohren durch Abpumpen erniedrigt und damit die Schicht wieder auf eine gleiche aber tiefere Temperatur $\left(60^{\circ} \mathrm{K}\right)$ gebracht. Nun wurden sowohl die Temperatur als auch der elektrische Widerstand der Schicht gemessen. Darauf wurde im Trägerrohr I Normaldruck hergestellt und nur das Trägerrohr II weiter abgepumpt. Im nunmehr symmetrisch umgekehrten Temperaturfeld wurde erneut die Thermospannung gemessen.

Zuletzt wurde noch eine Kontrollmessung des elektrischen Widerstandes im Ausgangszustand durchgeführt, um sicherzustellen, daß während der eben beschriebenen Meßvorgänge weder Strukturveränderungen in der Schicht erfolgt waren, noch Fremdatome aus dem Restgas adsorbiert worden waren. Mit Ausnahme einiger weniger Fälle nahm der elektrische Widerstand seinen Ausgangswert an; lediglich bei Cs war er meist um etwa $1-2 \%$ kleiner. Offenbar waren bei Cs die Ordnungsvorgänge in der Schicht nach Beendigung des Aufdampfvorganges noch nicht restlos abgelaufen.

Nach Beendigung einer solchen Meßreihe, die etwa 60-70 Minuten dauerte, wurde der Atomstrahl wieder freigegeben und durch weiteres Aufdampfen eine dikkere Schicht hergestellt, an der die eben beschriebenen Messungen wiederholt wurden.

16 Schrifttumsnachweis bei H. MAYER ${ }^{17}$.

17 H. MAyer, Physik dünner Schichten, Bd. I., Wiss. Verlagsges., Stuttgart 1950.

18 Sh. Datz u. E. H. TAylor, J. Chem. Phys. 25, 389 [1956]. 


\section{Die mittlere freie Weglänge der Leitungselektronen in $\mathrm{K}$, $\mathrm{Rb}$ und $\mathrm{Cs}$}

\section{Der Einfluß der Grenzflächen auf den elektrischen Widerstand}

Für höhere Temperaturen als die Debye-Temperatur kann der spezifische elektrische Widerstand $\varrho_{\infty}$ eines unendlich ausgedehnten Metalles durch die Beziehung ${ }^{1,2}$

$$
\varrho_{\infty}=\frac{m^{*} v}{\varepsilon^{2} N} \frac{1}{l_{\infty}}=\frac{\sqrt{2 m^{*} \zeta}}{\varepsilon^{2} N} \frac{1}{l_{\infty}}
$$

ausgedrückt werden. Darin bedeuten $m^{*} v$ den Impuls der Leitungselektronen an der Fermi-Kante $\zeta$, $\varepsilon$ die Elementarladung, $N$ die Elektronenkonzentration und $l_{\infty}$ die mittlere freie Weglänge.

Bei dünnen Schichten wird durch die Wechselwirkung der Elektronen mit den Grenzflächen die mittlere freie Weglänge verkürzt und damit eine Erhöhung des elektrischen Widerstandes hervorgerufen. Bezeichnet man mit $\varrho$ den spezifischen elektrischen Widerstand einer Schicht und mit $\varrho_{\infty}$ den des unendlich ausgedehnten Metalles gleicher Struktur wie die Schicht, so ist das Verhältnis $\varrho / \varrho_{\infty}$ eine Funktion von $l_{\infty} / d$, wenn mit $d$ die Schichtdicke bezeichnet wird. Wenn $l_{\infty} / d<1$ ist, läßt sich die Funktion

$$
\varrho / \varrho_{\infty}=f\left(l_{\infty} / d\right)
$$

in eine TAyLor-Reihe nach steigenden Potenzen von $l_{\infty} / d$ entwickeln und nach dem ersten Glied abbrechen. Weil $f(0)=1$ sein $\mathrm{mu} \beta$, folgt:

$$
\varrho / \varrho_{\infty}=1+\left(l_{\infty} / d\right) \cdot f^{\prime}(0) .
$$

Eine genaue Rechnung ${ }^{8,19-21}$ zeigt, da $f^{\prime}(0)=$ $3(1-p) / 8$ ist, wobei $p$ der Anteil der Leitungselektronen ist, deren Wechselwirkung mit der Grenzfläche ohne Energieübertragung erfolgt. Verschiedene Versuche, $p$ experimentell zu bestimmen oder abzuschätzen, gelangen bisher nur mit Hilfe zusätzlicher Hypothesen 22-24. Bei abgeschreckt kondensierten Schichten ist wegen des statistischen Charakters des Aufdampfvorganges die Annahme berechtigt, vorerst $p=0 \mathrm{zu}$ setzen.

In Abb. 2 ist der exakt berechnete theoretische Verlauf von $\varrho / \varrho_{\infty}$ nach Werten von SondHeimer ${ }^{8}$ dem der Näherungsformel (3) für dicke Schichten

19 R. G. Chambers, Nature, Lond. 165, 239 [1950].

20 R. G. Chambers, Proc. Roy. Soc., Lond. A 202, 378 [1950].

21 R. B. Dingle, Proc. Roy. Soc., Lond. A 201, 545 [1950].

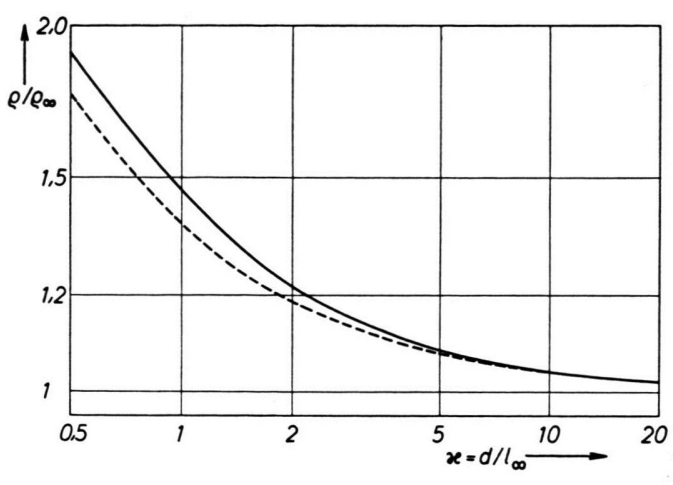

Abb. 2. Der theoretisch berechnete Verlauf des elektrischen Widerstandes als Funktion der Schichtdicke; die ausgezogene Kurve stellt den exakten Verlauf dar, die gestrichelte Kurve den nach der Näherungsformel berechneten.

gegenübergestellt. Man entnimmt diesem Vergleich, daß die Beziehung (3) im Bereich $l_{\infty} / d \leq 1$ gut anwendbar ist, da die Abweichungen weniger als $8 \%$ betragen.

Nach einfacher Umformung erhält man aus (3)

$$
\varrho d=\varrho_{\infty}\left(d+\frac{3}{8} l_{\infty}\right) \text {. }
$$

Daraus folgt, daß das Produkt der Meßgrößen $(\varrho d)$ gegen die Schichtdicke $d$ aufgetragen eine Gerade ergeben muß. Aus dem Schnitt dieser Geraden mit der $d$-Achse läßt sich $l_{\infty}$ und aus dem Schnitt mit der $(\varrho d)$-Achse das Produkt $\left(\varrho_{\infty} l_{\infty}\right)$ bestimmen.

Der so experimentell bestimmbare Wert von $\left(\varrho_{\infty} l_{\infty}\right)$ ist für die Elektronentheorie der Metalle von besonderer Bedeutung. Denn aus der Beziehung (1) ersieht man, daß $\left(\varrho_{\infty} l_{\infty}\right)$ bei Kenntnis von $m^{*}$, $\zeta, \varepsilon$ und $N$ auch berechnet werden kann; hierzu muß man für $m^{*}$ und $N$ gewisse Annahmen machen. Es liegt nun nahe, für Alkalimetalle versuchsweise die effektive Elektronenmasse $m^{*}$ gleich der Masse des freien Elektrons $m$, und für die Zahl der Leitungselektronen $N$ die Zahl der Atome pro Volumeneinheit $N_{A}$ zu setzen. Das Verhältnis des so theoretisch berechneten zu dem experimentell ermittelten Wert von $\left(\varrho_{\infty} l_{\infty}\right)$ ist dann gegeben durch:

$$
P=\frac{\left(\varrho_{\infty} l_{\infty}\right)_{\text {th. }}}{\left(\varrho_{\infty} l_{\infty}\right)_{\text {exp. }}}=\frac{N}{N_{A}} \sqrt{\frac{m}{m^{*}}}
$$

und bildet ein Maß für die Abweichungen von den gemachten Annahmen.

22 W. Cirkler, Z. Phys. 147, 481 [1957].

23 J. Niebuhr, Z. Phys. 132, 468 [1952].

24 D. G. Worden u. G. C. Danielson, J. Phys. Chem. Solids 6, 89 [1958]. 


\section{Meßergebnisse}

Die experimentelle Grundlage für die Bestimmung der mittleren freien Weglänge und des Produktes $\left(\varrho_{\infty} l_{\infty}\right)$ bildet der Verlauf des spezifischen elektrischen Widerstandes $\varrho$ in Abhängigkeit von der Schichtdicke $d$, wie er in Abb. 3 für K, Rb und Cs bei $90^{\circ} \mathrm{K}$ dargestellt ist. Die Meßpunkte entstammen verschiedenen Meßreihen. Meßpunkte derselben Meßreihe sind mit dem gleichen Zeichen markiert, um einen Überblick über die Reproduzierbarkeit der Meßpunkte zu geben.

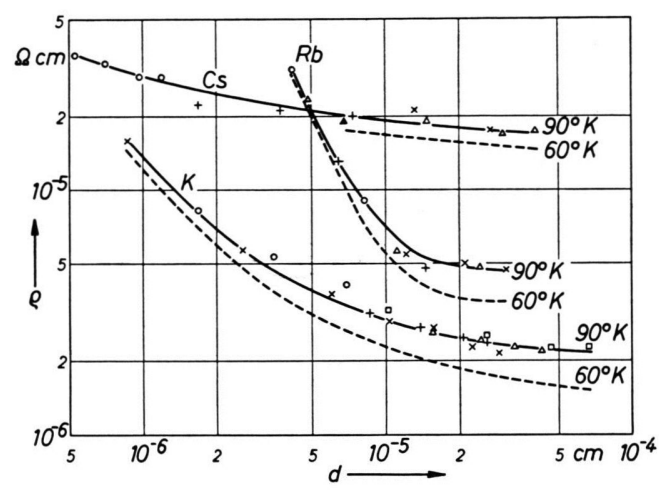

Abb. 3. Der spezifische elektrische Widerstand von K-, Rbund Cs-Schichten als Funktion der Schichtdicke bei $90^{\circ} \mathrm{K}$ und $60^{\circ} \mathrm{K}(---)$.

Auf diese Meßreihen wurde nun die Beziehung (4) angewendet und das Produkt ( $\varrho d)$ als Funktion der Schichtdicke $d$ aufgetragen. Man entnimmt der Abb. 4, daß entsprechend der theoretischen Erwartung für jedes der drei Metalle das Produkt $(\varrho d)$ als eine lineare Funktion der Schichtdicke $d$ darstellbar ist.

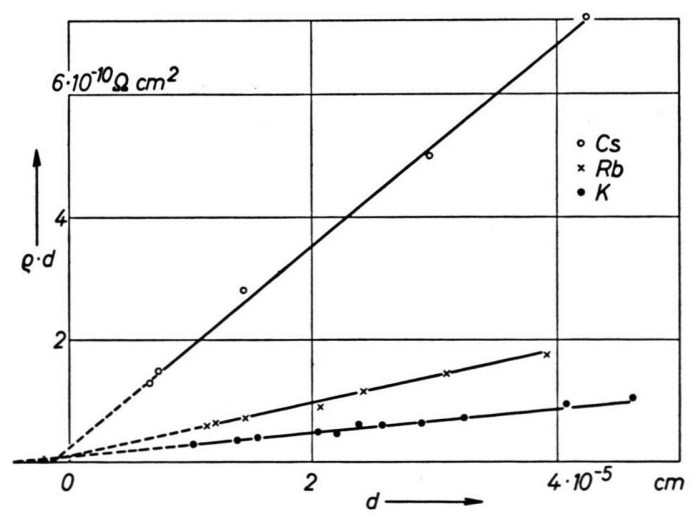

Abb. 4. Das Produkt $(\varrho d)$ bei $90{ }^{\circ} \mathrm{K}$ als Funktion der Schichtdicke für K, Rb und Cs.

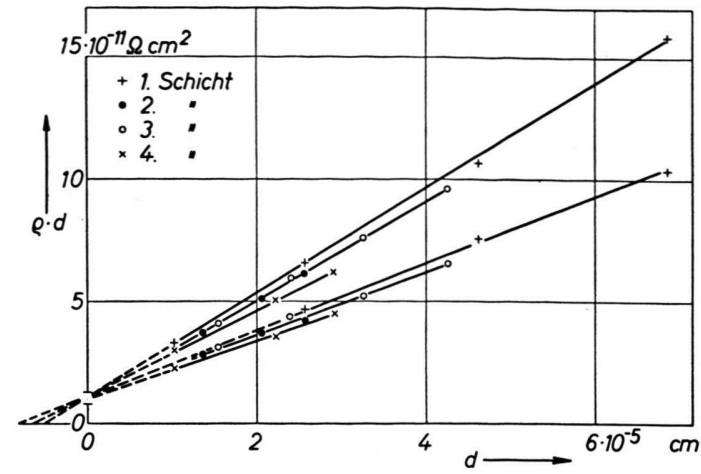

Abb. 5. Das Produkt $(\varrho d)$ bei $90^{\circ} \mathrm{K}$ und bei $60^{\circ} \mathrm{K}$ als Funktion der Schichtdicke für vier verschiedene Kaliumschichten.

Um die Streuung der Meßwerte von Schicht zu Schicht aufzuzeigen, ist in Abb. 5 der Verlauf der Geraden von 4 an verschiedenen Tagen aufgedampften Kaliumschichten für $90^{\circ} \mathrm{K}$ und $60^{\circ} \mathrm{K}$ aufgezeichnet. Die zur selben Schichte gehörigen Meßwerte weichen kaum von einer Geraden ab. Die verschiedenen Geraden schneiden jedoch die Ordinate im selben Punkt. Die Beziehung (4) beschreibt also innerhalb ihres Gültigkeitsbereiches das Verhalten des elektrischen Widerstandes dünner Schichten richtig. Dies berechtigt die Geraden zur numerischen Auswertung von $l_{\infty}$ und $\left(\varrho_{\infty} l_{\infty}\right)$ heranzuziehen. Die Ergebnisse sind in Tab. 1 zusammengestellt.

$\mathrm{Zu}$ den in Tab. 1 angegebenen statistischen Fehlern kommen noch systematische Fehler hinzu. So wurde bei der Schichtdickebestimmung der Wert des Ionisierungskoeffizienten von Datz und TAYLOR benutzt, ohne daß der Fehler, mit dem diese Messungen behaftet sind, berücksichtigt wurde. Eine weitere systematische Fehlerquelle liegt in der Ungenauigkeit, mit der die Länge und die Breite der Schicht $\mathrm{zu}$ messen sind. Die Schichtbreite ist wegen des "Halbschatteneffektes" der Blenden mit einem maximalen Fehler von $7 \%$ behaftet. Weil die Länge der Schicht aus experimentellen Gründen nur 6 bis $9 \mathrm{~mm}$ betragen konnte und die Spannungskontakte eine Mindestbreite von $\sim 1 \mathrm{~mm}$ haben mußten, um Kontaktschwierigkeiten sicher auszuschließen, konnte die Schichtlänge nur mit einem großen Fehler bestimmt werden; er betrug maximal 17\%. Angesichts dieser hohen systematischen Fehler wurde bei der Berechnung des spezifischen Widerstandes die Änderung der Geometrie infolge thermischer Ausdehnung nicht mehr berücksichtigt.

Vergleicht man die gemessenen Werte von $\left(\varrho_{\infty} l_{\infty}\right)$ mit den theoretisch berechneten, so fällt auf, daß die 


\begin{tabular}{|c|c|c|c|c|c|c|c|}
\hline & $\begin{array}{l}\left(l_{\infty}\right)_{90 \circ \mathbf{K}} \\
\cdot 10^{5}(\mathrm{~cm})\end{array}$ & $\begin{array}{l}\left(l_{\infty}\right)_{60 \circ \mathrm{K}} \\
\cdot 10^{5}(\mathrm{~cm})\end{array}$ & $\begin{array}{l}\left(\varrho_{\infty} \cdot l_{\infty}\right)_{\text {exp. }} \\
\cdot 10^{11}\left(\Omega \cdot \mathrm{cm}^{2}\right)\end{array}$ & $\begin{array}{l}\left(\varrho_{\infty} \cdot l_{\infty}\right)_{\mathrm{th} .} \\
\cdot 10^{11}\left(\Omega \cdot \mathrm{cm}^{2}\right)\end{array}$ & $P$ & $\frac{m^{*}}{m}$ & $\frac{N}{N_{A}}$ \\
\hline $\mathrm{K}$ & $1,23 \pm 0,22$ & $1,96 \pm 0,14$ & $\begin{array}{c}2,67 \underset{(0,8)}{ \pm 0,19} \\
\text { (0,8) }\end{array}$ & 2,25 & 0,84 & 0,94 & 0,81 \\
\hline $\mathrm{Rb}$ & $0,65 \pm 0,15$ & $0,86 \pm 0,19$ & $2,8 \underset{(0,6)}{ \pm} 0,3$ & 2,6 & 0,93 & 0,87 & 0,87 \\
\hline $\mathrm{Cs}$ & $0,40 \pm 0,09$ & $0,50 \pm 0,10$ & $6,8 \underset{(4,3)}{ \pm 0,7}$ & 2,9 & 0,43 & 0,83 & 0,39 \\
\hline
\end{tabular}

Tab. 1.

Übereinstimmung für $\mathrm{Rb}$ innerhalb der statistischen Fehlergrenzen liegt. Bei $\mathrm{K}$ ist die Übereinstimmung nicht mehr so gut, aber immerhin noch innerhalb der systematischen Fehlergrenzen. Bei Cs liegt sie jedoch außerhalb aller Fehlergrenzen, was soviel bedeutet, daß hier die Voraussetzungen bei der Berechnung von $\left(\varrho_{\infty} l_{\infty}\right)$ nicht mehr erfüllt sind.

Zum Vergleich sind in den Klammern die Werte eingetragen, die sich aus den Messungen von LovelL ${ }^{26}$ unter Verwendung der Beziehung (4) ergeben. Bei Kalium und Rubidium liegen die Werte in schlechter Übereinstimmung sowohl mit den Ergebnissen der vorliegenden Arbeit als auch mit den theoretischen Werten. Lediglich bei Caesium könnte man von einer besseren Übereinstimmung sprechen. Dies bedeutet aber wieder nur, daß bei Caesium die theoretischen Voraussetzungen nicht erfüllt sind.

Berücksichtigt man das Verhältnis $m^{*} / m$, von BRooks berechnet und von KItTeL ${ }^{27}$ mitgeteilt, so läßt sich das Verhältnis der Leitungselektronen zur Zahl der Atome berechnen. Man entnimmt der letzten Spalte, daß bei Rb jedes Atom mit einem Elektron zur Leitfähigkeit beiträgt. Bei $\mathrm{K}$ dürfte diese Annahme auch noch knapp erfüllt sein. Hingegen bei Cs entfällt ein Leitungselektron erst auf zwei Atome.

Um zu prüfen, bis zu welchen Schichtdicken die Weglängentheorie das Verhalten des elektrischen Widerstandes richtig beschreibt, wurde mit den Werten der Tab. 1 für $90^{\circ} \mathrm{K}$ das Verhältnis $\varrho / \varrho_{\infty}$ als Funktion von $\varkappa=d / l_{\infty}$ berechnet und mit der theoretischen Kurve, die nach Werten von SondHeimer ${ }^{8}$ in Abb. 6 ausgezogen eingezeichnet ist, verglichen. Bei Kalium liegen die Meßpunkte bis herab zu $170 \AA$ noch in guter UUbereinstimmung mit der theoretischen Kurve. Nach dünneren Schichten hin steigt jedoch $\varrho / \varrho_{\infty}$ stärker an als es die Theorie erwartet.

25 Vgl. Ch. Kittel ${ }^{27}$, S. 313.

26 A. C. B. Lovell, Proc. Roy. Soc., Lond. A 166, 270 [1938].

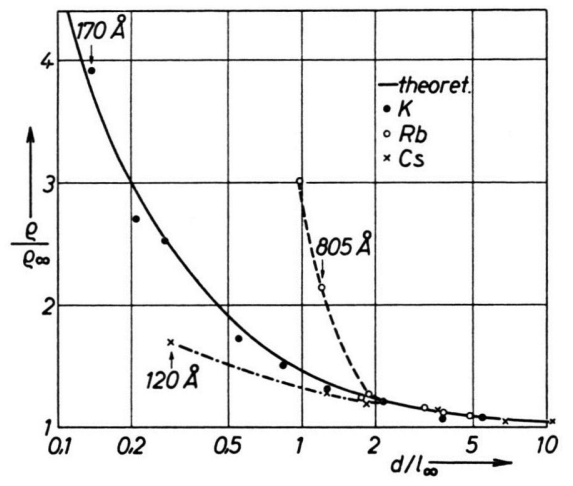

Abb. 6. Vergleich des experimentell festgestellten Verlaufs des elektrischen Widerstandes mit dem von der Theorie berechneten.

Diese Abweichung ist jedoch in diesem Dickebereich zu erwarten, weil hier die Bedingung der Planparallelität der Schichten wegen des statistischen Aufdampfvorganges und der endlichen Größe der Atome nicht mehr erfüllt ist.

Bei Rubidium und Caesium liegen die Verhältnisse nicht so günstig. Bei $\mathrm{Rb}$ weichen die Meßpunkte bereits bei einer Dicke von etwa $1100 \AA$ im selben Sinn wie bei $\mathrm{K}$ ab. Cs hingegen zeigt erst unterhalb von $600 \AA$ eine Abweichung, die aber in gegensinniger Weise wie bei $\mathrm{K}$ verläuft. Diese $\mathrm{Ab}$ weichungen mit einer viel größeren Oberflächenrauhigkeit als bei $\mathrm{K}$ zu erklären erscheint jedoch unbefriedigend.

\section{Die Matthiessensche Regel im Bereich dünner Schichten}

\section{Theoretischer Überblick}

Um die Abhängigkeit der mittleren freien Weglänge von der Energie der Leitungselektronen nachzuprüfen, ist es erforderlich, die dem Idealwider-

27 Ch. Kittel, Introduction à la physique de l'état solide, Dunod, Paris 1958. 
stand $\varrho_{\mathrm{i}}$ zuzuordnende mittlere freie Weglänge $l_{\mathrm{i}}$ und die dem Restwiderstand $\varrho_{\mathrm{r}}$ zuzuordnende mittlere freie Weglänge $l_{\mathrm{r}}$ zu kennen. Aus der Beziehung (1), die für ein Metall mit einem Leitungsband sowohl für den Idealwiderstand als auch für den Restwiderstand gültig ist, folgt:

$$
\varrho_{\infty} l_{\infty}=\varrho_{\mathrm{i}} l_{\mathrm{i}}=\varrho_{\mathrm{r}} l_{\mathrm{r}} .
$$

Zieht man die Matthiessensche Regel $\varrho_{\infty}=\varrho_{\mathrm{i}}+\varrho_{\mathrm{r}}$ hinzu, so ergibt sich aus diesen beiden Beziehungen:

$$
1 / l_{\infty}=1 / l_{\mathrm{i}}+1 / l_{\mathrm{r}} \text {. }
$$

Nun haben bereits einige Autoren ${ }^{23,26,28}$ an dünnen Schichten für $\mathrm{d} \varrho / \mathrm{d} T$ den Wert des massiven Metalles gefunden. Das bedeutet aber, daß in dünnen Schichten und im kompakten Metall $\varrho_{\text {i }}$ gleich ist. Folglich darf man aus dem am massiven Metall gemessenen Restwiderstand den Idealwiderstand $\varrho_{\mathrm{i}}$ bestimmen und mit den Beziehungen (6) und (7) $l_{\mathrm{i}}$ und $l_{\mathrm{r}}$ berechnen.

Da aber nicht bekannt ist, ob diese Ergebnisse für beliebig dünne Schichten auch noch zutreffen und ob sie mit der Weglängentheorie verträglich sind, ist es erforderlich, diese Frage zunächst theoretisch und experimentell zu untersuchen.

Durch logarithmische Differentiation der Beziehungen (1) und (2) nach der Temperatur erhält man:

$$
\begin{aligned}
\frac{1}{\varrho_{\infty}} \frac{\mathrm{d} \varrho_{\infty}}{\mathrm{d} T} & =-\frac{1}{l_{\infty}} \frac{\mathrm{d} l_{\infty}}{\mathrm{d} T}-\frac{1}{N} \frac{\mathrm{d} N}{\mathrm{~d} T}, \\
\frac{1}{\varrho} \frac{\mathrm{d} \varrho}{\mathrm{d} T} & =\frac{1}{\varrho_{\infty}} \frac{\mathrm{d} \varrho_{\infty}}{\mathrm{d} T}+\frac{z}{f} \frac{\mathrm{d} f}{\mathrm{~d} z} \frac{1}{l_{\infty}} \frac{\mathrm{d} l_{\infty}}{\mathrm{d} T} ; \quad z=\frac{l_{\infty}}{d} .
\end{aligned}
$$

Dabei wurde berücksichtigt, daß die Elektronenkonzentration $N$ im kompakten Metall eventuell von der Temperatur abhängen könnte. Hingegen wurde die Änderung der Schichtdicke infolge der Temperaturvariation vernachlässigt. Elimination von $\left(1 / l_{\infty}\right) \cdot\left(\mathrm{d} l_{\infty} / \mathrm{d} T\right)$ aus diesen beiden Gleichungen führt unter Berücksichtigung von (2) zu

$$
\frac{\mathrm{d} \varrho}{\mathrm{d} T}=\frac{\mathrm{d} \varrho_{\infty}}{\mathrm{d} T}\left[f(z)-z\left(1+\frac{\mathrm{d} N}{N} / \frac{\mathrm{d} \varrho_{\infty}}{\varrho_{\infty}}\right) \frac{\mathrm{d} f}{\mathrm{~d} z}\right] .
$$

Die Matthiessensche Regel ist somit in jenem Schichtdickebereich gültig, in dem

$$
f(z)-z\left(1+\frac{\mathrm{d} N}{N} / \frac{\mathrm{d} \varrho_{\infty}}{\varrho_{\infty}}\right) \frac{\mathrm{d} f}{\mathrm{~d} z}=1
$$

ist. Da $\frac{\mathrm{d} N}{N} / \frac{\mathrm{d} \varrho_{\infty}}{\varrho_{\infty}}=b$ nur von der Temperatur, aber

${ }^{28}$ F. W. Reynolds u. G. R. Stilwell, Phys. Rev. 88, 418 [1952]. nicht von der Schichtdicke abhängt, erhält man als Lösung dieser Differentialgleichung

$$
\frac{\varrho}{\varrho_{\infty}}=f(z)=1+c\left(\frac{l_{\infty}}{d}\right)^{1 /(1+b)} .
$$

Wenn sich die Elektronenkonzentration $\mathrm{d} N / N$ im untersuchten Temperaturintervall nur sehr wenig oder gar nicht ändert, kann $b$ vernachlässigt werden. Die Beziehung (10) führt dann mit der Integrationskonstanten $c=3 / 8$ zu dem Ausdruck (4), mit dem die Weglängentheorie das Widerstandsverhalten dicker Schichten beschreibt. Die Matthiessensche Regel ist daher mit der Weglängentheorie im Bereich dicker Schichten $d \geq l_{\infty}$ verträglich. Die Abweichungen von der Matthiessenschen Regel im Bereich dünnster Schichten $d \leq l_{\infty}$ werden durch die Beziehung (9) beschrieben und sind in Abb. 7 für verschiedene Werte von $b$ graphisch dargestellt. Dieser

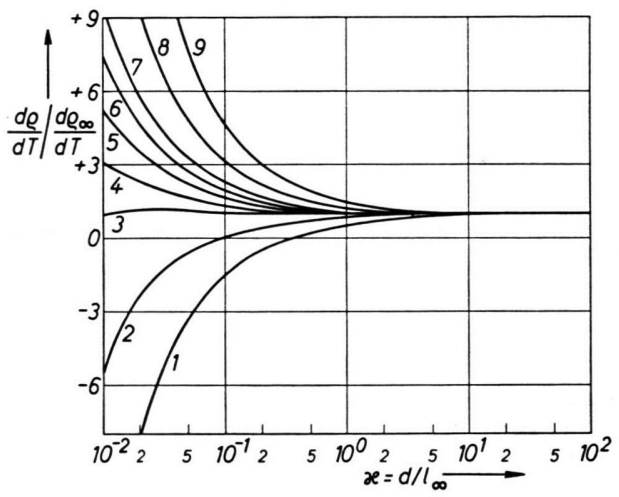

Abb. 7. Der theoretische Verlauf der durch die Temperatur bedingten Widerstandsänderung als Funktion der Schichtdicke für verschiedene Werte des Parameters $b$.

$\begin{array}{llllllllll}\text { Kurve } & 1 & 2 & 3 & 4 & 5 & 6 & 7 & 8 & 9\end{array}$ $b=\quad+1+0,5+0,2+0,1+0-0,1-0,2-0,5-1$

Darstellung entnimmt man, daß im Bereich ausreichend dicker Schichten für jeden beliebigen Wert von $b, \mathrm{~d} \varrho / \mathrm{d} T=\mathrm{d} \varrho_{\infty} / \mathrm{d} T$ sein sollte. Wenn nun auch das Experiment dieses Ergebnis bestätigt, kann $l_{\mathrm{i}}$ und $l_{\mathrm{r}}$ bestimmt werden.

\section{Meßergebnisse}

Aus den bei $90{ }^{\circ} \mathrm{K}$ und $60{ }^{\circ} \mathrm{K}$ durchgeführten Messungen wurde $\Delta \varrho / \Delta T$ als Funktion der Schichtdicke ermittelt. Das in Abb. 8 dargestellte Ergebnis zeigt, wie $\Delta \varrho / \Delta T$ mit wachsender Dicke dem Wert des massiven Metalles als Grenzwert zustrebt. Das theoretisch zu erwartende Ergebnis

$$
\left(\mathrm{d} \varrho_{\infty} / \mathrm{d} T\right)_{\text {Schicht }}=\left(\mathrm{d} \varrho_{\infty} / \mathrm{d} T\right)_{\text {mass. Metall }} \text { für } d \geqq l_{\infty}
$$




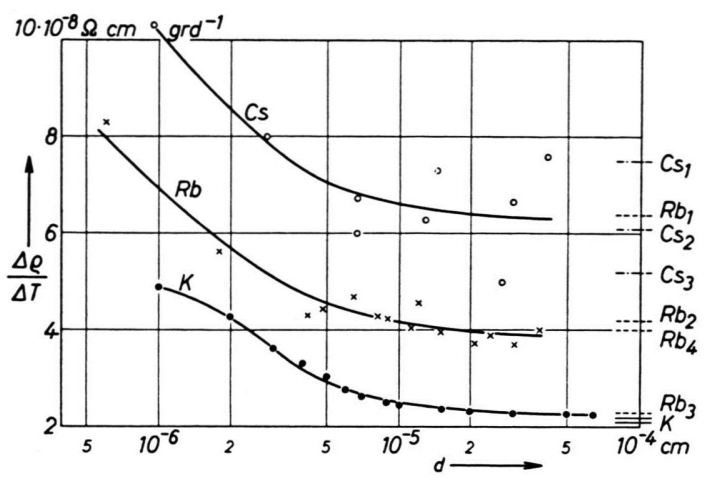

Abb. 8. Der experimentelle Verlauf der thermischen Widerstandsänderung im Bereich $60^{\circ} \mathrm{K}$ bis $90^{\circ} \mathrm{K}$ als Funktion der Schichtdicke für $\mathrm{K}, \mathrm{Rb}$ und $\mathrm{Cs}$. Am rechten Rand die am kompakten Metall gemessenen Werte verschiedener Autoren.

ist allerdings nur für Kalium experimentell eindeutig bestätigt, weil die am massiven Metall gefundenen Werte von McLennan und Niven ${ }^{29}$ sowie von Woltjer und Kamerlingh OnNes ${ }^{30}$ praktisch identisch und gleich dem gefundenen Grenzwert der Schichten sind. Bei $\mathrm{Rb}$ und $\mathrm{Cs}$ hingegen weichen die Ergebnisse der verschiedenen Autoren stark voneinander ab, wie dies aus den rechts am Rande der Abb. 8 eingezeichneten Werten zu erkennen ist. Aus den relativen Widerstandsmessungen von Meissner und VorgT ${ }^{31}$ erhält man für $\mathrm{Rb}$ und $\mathrm{Cs}$ die mit $\mathrm{Rb}_{1}$ bzw. mit $\mathrm{Cs}_{1}$ markierten Werte, sofern man aus der von ihnen angegebenen Geometrie den spezifischen Widerstand berechnet und verwendet, der aber viel größer als der von anderen Autoren gefundene Wert ist. Verwendet man für $\varrho_{\infty}$ den Tabellenwert ${ }^{32}$, so erhält man aus ihren Messungen des Widerstandes die mit $\mathrm{Rb}_{2}$ bzw. mit $\mathrm{Cs}_{2}$ bezeichneten Werte. Die mit dem Index 3 versehenen Werte sind den Messungen von $\mathrm{H}_{\mathrm{ACKSPILL}}{ }^{33}$ entnommen und der Wert $\mathrm{Rb}_{4}$ wurde von McLennan und Niven ${ }^{29}$ mitgeteilt. Trotz dieser großen Streuung scheint die Gültigkeit der Beziehung (11) auch für $\mathrm{Rb}$ und $\mathrm{Cs}$ sehr wahrscheinlich zu sein.

Da das Experiment die Beziehung (11) bestätigte, wurde der Idealwiderstand $\varrho_{\mathrm{i}}$ aus den Tabellenwerten ${ }^{32}$ für den Rest- und den Gesamtwiderstand berechnet, ohne jedoch zu berücksichtigen, daß nach Henning ${ }^{34}$ der Restwiderstand streng genommen keine additive Größe ist. Mit Hilfe der Gln. (6) und

29 J. C. McLennan u. C. D. Niven, Phil. Mag. (7) 4, 386 [1927].

30 H. R. Woltuer u. H. Kamerlingh Onnes, Comm. Kammerlingh Onnes Lab. Leiden, 173 a, 3 [1924].
(7) erhält man sofort die Werte für $l_{\mathrm{i}}$ und $l_{\mathrm{r}}$. Ferner kann man aus dem Verhältnis $l_{\mathrm{i}} / l_{\mathrm{r}}=\varrho_{\mathrm{r}} / \varrho_{\mathrm{i}}$ auf die Konzentration der Gitterfehler in der Schicht schließen. In der Tab. 2 ist das Ergebnis für $90{ }^{\circ} \mathrm{K}$ zusammengefaßt.

\begin{tabular}{|l|l|l|l|}
\hline & \multicolumn{1}{|c|}{$\mathrm{K}$} & $\mathrm{Rb}$ & $\mathrm{Cs}$ \\
\hline$l_{\mathrm{i}} \cdot 10^{5}(\mathrm{~cm})$ & 1,6 & 0,87 & 1,16 \\
$l_{\mathrm{r}} \cdot 10^{5}(\mathrm{~cm})$ & 5,2 & 3,1 & 0,66 \\
$\left(l_{\mathrm{i}} / l_{\mathrm{r}}\right)_{\text {Schicht }}$ & 0,31 & 0,28 & 1,8 \\
$\left(\varrho_{\mathrm{r}} / \varrho_{\mathrm{i}}\right)_{\text {mass. Metall }}$ & 0,027 & 0,12 & 0,13 \\
\hline
\end{tabular}

Tab. 2.

Bemerkenswert ist, daß die Konzentration der Gitterfehler in den Schichten - mit Ausnahme von $\mathrm{Rb}$ - mehr als das 10-fache der üblicherweise im kompakten Metall vorhandenen beträgt.

Die Beziehung (11) führt ferner zu einer neuen Methode die Schichtdicke zu bestimmen. Aus $R=\varrho c / d$, worin $c$ das Verhältnis von Schichtlänge zur Breite und $R$ den elektrischen Widerstand der Schicht bezeichnen, folgt:

$$
d=c(\Delta \varrho / \Delta T) /(\Delta R / \Delta T) \text {. }
$$

Da $c$ unmittelbar gemessen werden kann und für $\Delta \varrho / \Delta T$ nach (11) der Wert des kompakten Metalles eingesetzt werden darf, braucht nur der elektrische Widerstand bei zwei bekannten Temperaturen gemessen zu werden, um $\Delta R / \Delta T$ und damit $d$ zu bestimmen. Dabei muß aber beachtet werden, daß keinerlei Strukturänderungen in der Schicht während der Messung stattfinden, und daß die Schicht ausreichend dick $\left(d>l_{\infty}\right)$ ist.

Im Bereich dünnster Schichten nimmt $\Delta \varrho / \Delta T$ mit abnehmender Schichtdicke zu (Abb. 8). Für den quantitativen Vergleich mit der Theorie wurde der Quotient $(\Delta \varrho / \Delta T) /\left(\Delta_{\infty} / \Delta T\right)$ gegen $d / l_{\infty}$ aufgetragen, wobei die mittlere freie Weglänge $l_{\infty}$ auf die Zwischentemperatur von $75^{\circ} \mathrm{K}$ bezogen wurde. Die ausgezogenen Kurven in Abb. 9 stellen den nach (9) berechneten Verlauf für $b=0$ und $b=-0,2$ dar. Alle Meßpunkte liegen jedoch oberhalb der theoretischen Kurve für $b=0$. Da die theoretischen Kurven für $p=0$ berechnet wurden, läge es nahe, diese $\mathrm{Ab}$ weichung auf einen Bruchteil der an den Grenzflä-

\footnotetext{
31 W. Meissner u. B. Voigt, Ann. Phys., Lpz. (5) 7, 761 [1930].

32 Landolt-Börnstein, 6. Aufl. Bd. II. 1959.

33 L. Hackspill, C. R. Acad. Sci., Paris 151, 305 [1910].

34 F. Henning, Ann. Phys., Lpz. (4) 40, 635 [1913].
} 


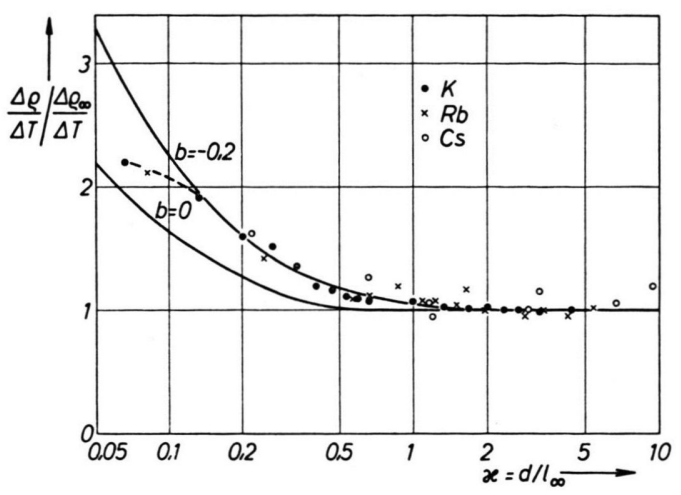

Abb. 9. Die thermische Widerstandsänderung von K-, Rbund Cs-Schichten als Funktion der Schichtdicke. Die ausgezogenen Kurven entsprechen dem nach der Theorie berechneten Verlauf für $b=0$ und $b=-0,2$.

chen „elastisch reflektierten“ Elektronen $(p \neq 0)$ zurückzuführen. Mit zunehmendem Anteil „elastisch reflektierter" Elektronen müßte jedoch der Weglängeneffekt kleiner werden und die Meßpunkte müßten unterhalb der theoretischen Kurve für $b=0$ liegen.

Als Ursache dieser Abweichung könnte aber eine Änderung der Konzentration der Leitungselektronen im untersuchten Temperaturintervall angesehen werden, weil die Meßpunkte recht gut mit der theoretischen Kurve für $b=-0,2$ zusammenfallen. Da $b=(\Delta N / N) /\left(\Delta \varrho_{\infty} / \varrho_{\infty}\right)$ gesetzt wurde, läßt sich die relative Änderung $\mathrm{d} N / N$ der Leitungselektronen leicht aus den Meßwerten berechnen. Man findet, daß die Zahl der Leitungselektronen bei $75^{\circ} \mathrm{K}$ pro Grad um 2,6\% bei K, um 2,0\% bei Rb und um $0,82^{\%} \%$ bei Cs mit sinkender Temperatur zunimmt. Da die Debye-Temperatur für $\mathrm{K}$ bei $99^{\circ} \mathrm{K}$, für $\mathrm{Rb}$ bei $59{ }^{\circ} \mathrm{K}$ und für $\mathrm{Cs}$ bei $43^{\circ} \mathrm{K}$ liegt, drängt sich die Vermutung auf, daß die Konzentration der Leitungselektronen in der Nähe dieser charakteristischen Temperatur Schwankungen unterworfen ist.

\section{Die absolute Thermokraft dïnner Schichten}

Die Wechselwirkung der Elektronen mit den Grenzflächen hat bei dünnen Schichten auch auf die absolute Thermokraft einen beachtlichen Einfluß, den man ausnützen kann, um die für quasifreie Elektronen vorhergesagte Beziehung: $l_{\mathrm{i}}=a E^{2} \mathrm{zu}$ prüfen. Der quantitative Zusammenhang zwischen absoluter Thermokraft und Schichtdicke, von Justi,

35 F. SAvornin, C. R. Acad. Sci., Paris 248, 2458 [1959].

38 F. Savornin, C. R. Acad. Sci., Paris 248, 3133 [1959].
Kohler und Lautz ${ }^{3,4}$ sowie von Savornin ${ }^{35,36}$ untersucht, wird durch

$\varphi-\varphi_{\infty}=\frac{1}{\varepsilon} \frac{\pi^{2}}{3} \frac{k^{2} T}{\zeta}\left(\frac{\mathrm{d} \ln l_{\infty}}{\mathrm{d} \ln E}\right)_{E=\zeta}\left(1-\frac{\varrho_{\infty}}{\varrho} \frac{\mathrm{d} \varrho}{\mathrm{d} T} / \frac{\mathrm{d} \varrho_{\infty}}{\mathrm{d} T}\right)$

ausgedrückt. Darin wurde mit $\varphi$ die absolute Thermokraft der dünnen Schicht und mit $\varphi_{\infty}$ die des unendlich ausgedehnten Metalles gleicher Struktur wie die Schicht bezeichnet. $k$ ist die BoltzmannKonstante, während die anderen Größen die bisher verwendeten Symbole tragen.

Experimentell wird jedoch die auf ein anderes Metall bezogene Thermospannung $\psi$ gemessen, welche die Differenz aus der absoluten Thermokraft $\varphi^{*}$ des Bezugsmetalles und der absoluten Thermokraft $\varphi$ des zu untersuchenden Metalles ist. Es gilt also $\psi=\varphi-\varphi^{*}$ und $\psi_{\infty}=\varphi_{\infty}-\varphi^{*}$, woraus

$$
\varphi-\varphi_{\infty}=\psi-\psi_{\infty}
$$

folgt.

Im Bereich dicker Schichten ist jedoch

$$
\mathrm{d} \varrho / \mathrm{d} T=\mathrm{d} \varrho_{\infty} / \mathrm{d} T .
$$

Unter Berücksichtigung der Beziehung (4) erhält man aus (13) :

$$
\begin{gathered}
\psi d=\psi_{\infty} d+\frac{1}{\varepsilon} \frac{\pi^{2}}{8} \frac{k^{2} T}{\zeta} l_{\infty}\left(\frac{\mathrm{d} \ln l_{\infty}}{\mathrm{d} \ln E}\right)_{E=\zeta} ; \\
d>l_{\infty} .
\end{gathered}
$$

Das Produkt der Meßgrößen $(\psi d)$ gegen die Schichtdicke $d$ aufgetragen muß eine Gerade ergeben, aus deren Schnitt mit der $d$-Achse $\psi_{\infty}$ und aus deren Schnitt mit der $(\psi d)$-Achse die Größe

$$
\frac{1}{\varepsilon} \frac{\pi^{2}}{8} \frac{k^{2} T}{\zeta} l_{\infty}\left(\frac{\mathrm{d} \ln l_{\infty}}{\mathrm{d} \ln E}\right)_{E=\zeta}
$$

bestimmt werden können. Da die mittlere freie Weglänge bereits ermittelt wurde, läßt sich $\left(\frac{d \ln l_{\infty}}{d \ln E}\right)_{E=\zeta}$ berechnen. Den Zusammenhang zwischen

$$
\left(\frac{\mathrm{d} \ln l_{\mathrm{i}}}{\mathrm{d} \ln E}\right)_{E=\zeta} \quad \text { und } \quad\left(\frac{\mathrm{d} \ln l_{\infty}}{\mathrm{d} \ln E}\right)_{E=\zeta}
$$

erhält man aus (7), wenn man berücksichtigt, daßs $l_{\mathrm{r}}$ von der Energie der Elektronen unabhängig ist. Eine einfache Rechnung führt zu:

$$
\left(\frac{\mathrm{d} \ln l_{\mathrm{i}}}{\mathrm{d} \ln E}\right)_{E=\zeta}=\frac{l_{\mathrm{i}}+l_{\mathrm{r}}}{l_{\mathrm{r}}}\left(\frac{\mathrm{d} \ln l_{\infty}}{\mathrm{d} \ln E}\right)_{E=\zeta} .
$$

Da $l_{\mathrm{i}}$ und $l_{\mathrm{r}}$ bestimmt werden konnten, läßt sich $\left(\frac{\mathrm{d} \ln l_{\mathrm{i}}}{\mathrm{d} \ln E}\right)_{E=\zeta} \quad$ ermitteln und damit die Aussage der Elektronentheorie der Metalle: $l_{\mathrm{i}}=a E^{2}$ nachprüfen. 


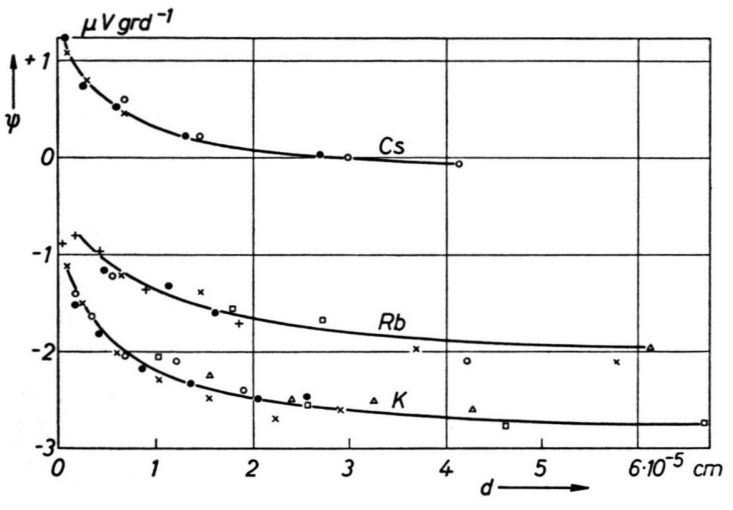

Abb. 10. Der experimentelle Verlauf der Thermospannung als Funktion der Schichtdicke.

In Abb. 10 ist die gemessene Thermospannung in Abhängigkeit von der Schichtdicke aufgetragen. Von diesen Meßwerten ausgehend, wurde das Produkt $(\psi d)$ gebildet und gegen die Schichtdicke aufgetragen. Die Meßwerte liegen für alle drei Alkalimetalle recht gut auf einer Geraden, in Übereinstimmung mit (14). Die quantitative Auswertung der Abb. 11 nach (14) und (15) wurde wieder auf

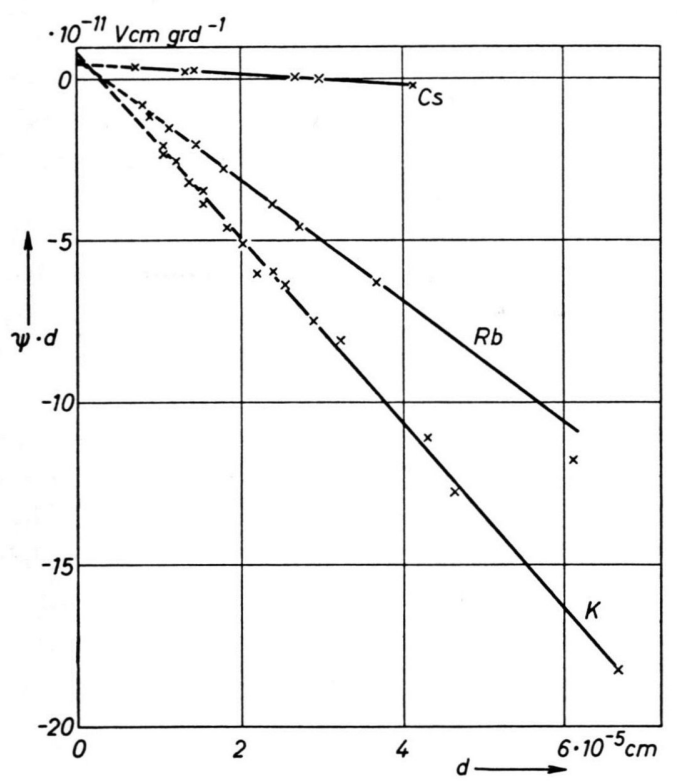

Abb. 11. Das Produkt $(w d)$ als Funktion der Schichtdicke.

$75{ }^{\circ} \mathrm{K}$ bezogen, der Temperatur, die in der Mitte zwischen der kalten und der warmen "Lötstelle“ liegt. Für die Fermische Grenzenergie $\zeta$ wurden die von Sommerfeld und Bethe ${ }^{1}$ mitgeteilten Werte eingesetzt. Das numerische Ergebnis zeigt Tab. 3.

\begin{tabular}{|c|r|r|c|}
\hline & $\mathrm{K}$ & $\mathrm{Rb}$ & $\mathrm{Cs}$ \\
\hline$\left(\frac{\mathrm{d} \ln l_{\infty}}{\mathrm{d} \ln E}\right)_{E=\zeta}$ & 1,7 & 2,1 & 2,5 \\
$\left(\frac{\mathrm{d} \ln l_{\mathrm{i}}}{\mathrm{d} \ln E}\right)_{E=\zeta}$ & 2,4 & 2,8 & 7,8 \\
$\psi \infty$ & $-2,9$ & $-1,9$ & $\begin{array}{c}-0,17 \\
\mu \mathrm{V} / \mathrm{grd}\end{array}$ \\
\hline
\end{tabular}

Tab. 3.

Es ist wegen der Einführung von $l_{\infty}, l_{\mathrm{i}}$ und $l_{\mathrm{r}}$ mit dem recht großen Fehler von etwa $50 \%$ behaftet. Innerhalb dieser Fehlergrenze werden für Kalium und Rubidium die Annahmen der Theorie bestätigt, die zur Abhängigkeit der mittleren freien Weglänge vom Quadrat der Energie der Leitungselektronen führen. Caesium hingegen zeigt ein stark abweichendes Verhalten. Das Modell der quasifreien Elektronen vermag die Verhältnisse bei diesem Metall nicht mehr richtig zu beschreiben. Da die Meßpunkte aber auch bei $\mathrm{Cs}$ auf einer Geraden liegen, muß $l_{\mathrm{i}}$ von der Energie der Leitungselektronen mit einer höheren Potenz als 2, nämlich 6 bis 9 , abhängen. $\psi_{\infty}$ ist bei allen drei Metallen rund um den Faktor drei kleiner als die am kompakten Metall von Bidwell ${ }^{37}$ oder Hackspill ${ }^{33,}{ }^{38}$ gemessenen Werte.

Um zu prüfen, bis zu welcher Schichtdicke die Weglängentheorie das thermoelektrische Verhalten der untersuchten Schichten richtig beschreibt, wur-

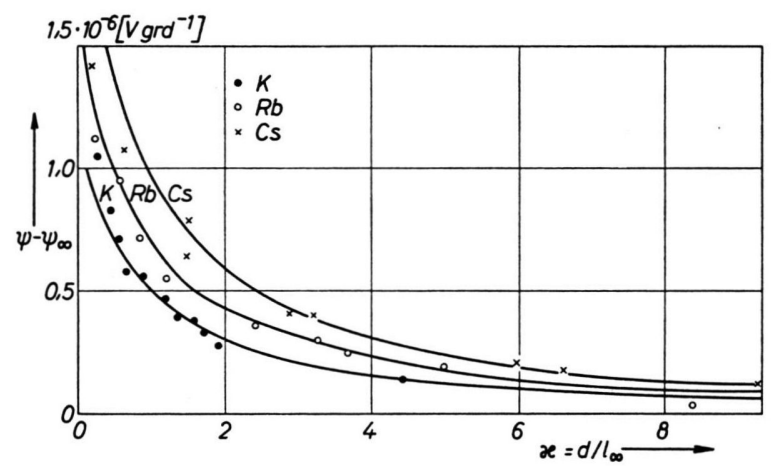

Abb. 12. Die Thermospannung als Funktion der Schichtdicke. Die ausgezogenen Kurven stellen den nach Formel (13) berechneten Verlauf dar.

den in Abb. 12 die experimentellen Werte für $\psi-\psi_{\infty}$ gegen $d / l_{\infty}$ aufgetragen. Die ausgezogenen Kurven wurden nach Formel (13) berechnet. Bei Rb decken sich experimenteller und theoretischer Verlauf im

37 C. C. Bidwell, Phys. Rev. 23, 357 [1924].

38 L. Hackspill u. W. Broniewski, Ann. Chim. Phys. 29, 455 [1913]. 
gesamten untersuchten Bereich von $180 \AA$ bis zu $6000 \AA$. Bei $K$ und $\mathrm{Cs}$ verläuft die thermoelektrische Spannung im Schichtdickebereich $d \geq 600 \AA$ in Übereinstimmung mit der Weglängentheorie. Für Schichten unterhalb $600 \AA$ Dicke liegen die Meßwerte bei $\mathrm{K}$ oberhalb der theoretischen Kurve, bei $\mathrm{Cs}$ darunter. Eine einwandfreie Begründung für diese Abweichung läßt sich wegen der geringen Zahl der bisher auf diesem Gebiete durchgeführten Messungen noch nicht geben.

\section{Diskussion}

Die hier mit der Methode der dünnen Schichten durchgeführten Messungen machten es möglich, einerseits Aussagen der Weglängentheorie quantitativ zu prüfen, andererseits charakteristische Größen der Metalle, wie die mittlere freie Weglänge und ihre Abhängigkeit von der Energie der Leitungselektronen, zu bestimmen.

Der Vergleich der Experimente mit der Theorie der Weglängeneffekte zeigte, daß die Theorie im Bereich dickerer Schichten $\left(d \geq l_{\infty}\right)$ das festgestellte Verhalten der Metalle richtig beschreibt. Daher erstreckte sich die quantitative Auswertung der Messungen vornehmlich auf diesen Bereich. Im Bereich dünner Schichten $\left(d<l_{\infty}\right)$ wird die Übereinstimmung von Theorie und Experiment mit abnehmender Schichtdicke immer schlechter. Nach Unterschreiten einer bestimmten Schichtdicke ist die Theorie der Weglängeneffekte nicht mehr anwendbar. Die Ursache dieser Abweichungen dürfte wohl darin liegen, daß wesentliche Voraussetzungen, die in der Theorie der Weglängeneffekte gemacht wurden, im Experiment nicht mehr erfüllt werden.

Wegen der Art, wie die Schichten hergestellt worden sind, liegt es nahe, die von der Theorie gemachten Annahmen, daß die Schichten planparallel und homogen sind, an Hand der experimentellen Ergebnisse kritisch zu prüfen. Wegen des statistischen Charakters des Aufdampfvorganges muß mit einer Rauhigkeit der Schichten von einigen Atomdurchmessern gerechnet werden. Erst wenn die Schichtdicke groß gegen diese Rauhigkeit ist, kann man die Schichten als planparallel ansehen und die Dicke definieren ${ }^{39}$. Die Abweichungen bei Kalium könnten damit zufriedenstellend erklärt werden, weil hier die kritische Schichtdicke zwischen 150 und $200 \AA$ liegt;

29 R. Nossek, Z. Phys. 142, 321 [1955]. das sind etwa $30-40$ Atomlagen. Die Rauhigkeit würde in diesem Falle 3-4 Atomdurchmesser betragen. Bei Rubidium hingegen müßte eine Rauhigkeit von 20 Atomdurchmessern angenommen werden, um diese Deutung aufrechtzuerhalten. Dies erscheint jedoch unplausibel. Man muß deshalb auch die andere Annahme der Theorie, die der Homogenität der Schichten, prüfen. Diese Annahme bedeutet ja nicht nur, daß die Schichten in sich homogen sind, sondern daß Schichten verschiedener Dicke, die miteinander verglichen werden, die gleiche Homogenität, das heißt die gleiche Struktur, besitzen.

Nach der Art der Herstellung können die erzeugten Schichten keine Einkristalle sein; sie sind polykristallin. Wie groß die einzelnen Kriställchen im Durchschnitt werden, hängt von der Temperatur und der Oberflächenbeschaffenheit des Schichtträgers, sowie von der Aufdampfgeschwindigkeit und dem Metall ab. Die Größe der Kristallite wirkt aber bestimmend auf den Restwiderstand, weil ja die Korngrenzen als Gitterfehler wirksam sind. Solange eine Schicht nicht jene Dicke erreicht hat, oberhalb welcher sich die Durchschnittsgröße der Kristallite bei weiterem Aufdampfen nicht mehr ändert, ist sie im Sinne der Weglängentheorie nicht als homogen zu betrachten. Diese Auffassung würde die beobachteten Abweichungen des Experiments von der Theorie ausreichend verständlich machen.

Das spricht nicht gegen die Richtigkeit der Weglängentheorie. Völlige Übereinstimmung zwischen Theorie und Experiment darf man nur erwarten, wenn die in der Theorie gemachten Annahmen experimentell realisiert werden.

Der Bereich, innerhalb dessen dies geschehen ist, war ausreichend groß um die Übereinstimmung nachzuweisen und die Größen zu bestimmen, die für die Elektronentheorie der Metalle von Interesse sind. Das Ergebnis, daß das Verhalten von Kalium und Rubidium oberhalb der Debye-Temperatur mit dem einfachen Modell der quasifreien Elektronen recht gut zu erklären ist, daß hingegen Caesium bereits stark von diesem Modell abweicht, ist keine Überraschung, sondern eine Bestätigung dessen, was man bisher vermutet hat.

Herrn Prof. Dr. H. Mayer bin ich zu Dank verpflichtet für die Förderung dieser Arbeit und das große Interesse, das er in zahlreichen anregenden Diskussionen bekundet hat. Ebenso danke ich der Deutschen Forschungsgemeinschaft für die zur Verfügung gestellten Sachmittel und Leihgaben. 